\title{
FACT - TeV Flare Alerts Triggering Multi-Wavelength Observations
}

D. Dorner ${ }^{* a}$, M. L. Ahnen ${ }^{b}$, M. Balbo ${ }^{d}$, M. Bergmann ${ }^{a}$, A. Biland ${ }^{b}$, T. Bretz ${ }^{b 1}$, K. A. Brügge ${ }^{c}$, J. Buss ${ }^{c}$, S. Einecke ${ }^{c}$, J. Freiwald ${ }^{c}$, C. Hempfling ${ }^{a}$, D. Hildebrand ${ }^{b}$, G. Hughes ${ }^{b}$, W. Lustermann ${ }^{b}$, K. Mannheim ${ }^{a}$, K. Meier $^{a}$, S. Müller ${ }^{b}$, D. Neise ${ }^{b}$, A. Neronov ${ }^{d}$, M. Nöthe ${ }^{c}$, A.-K. Overkemping ${ }^{c}$, A. Paravac ${ }^{a}$, F. Pauss ${ }^{b}$, W. Rhode ${ }^{c}$, F. Temme ${ }^{c}$, J. Thaele ${ }^{c}$, S. Toscano ${ }^{d}$, P. Vogler ${ }^{b}$, R. Walter ${ }^{d}$, and A. Wilbert ${ }^{a}$

${ }^{a}$ Universität Würzburg, Institute for Theoretical Physics and Astrophysics

Emil-Fischer-Str. 31, 97074 Würzburg, Germany

${ }^{b}$ ETH Zurich, Institute for Particle Physics

Otto-Stern-Weg 5, 8093 Zurich, Switzerland

${ }^{c}$ TU Dortmund, Experimental Physics 5

Otto-Hahn-Str. 4, 44221 Dortmund, Germany

${ }^{d}$ University of Geneva, ISDC Data Center for Astrophysics

Chemin d'Ecogia 16, 1290 Versoix, Switzerland

1 also at RWTH Aachen

E-mail: dornereastro.uni-wuerzburg.de

Active galactic nuclei show variability on time scales ranging from minutes to decades. The radiation from these extreme objects spans many orders of magnitude along the whole electromagnetic spectrum. The spectral energy distribution shows two peaks, where for the subgroup of blazars the first peak is in the radio to X-ray regime, while the second peak is in the gamma-ray regime. Due to the extreme variability and the wide spectral range, simultaneous multi-wavelength observations are vital to understand the underlying physics. Furthermore, long-term monitoring is crucial to obtain an unbiased data sample.

While for the measurements of the low-energy peak, many instruments are available, the data at $\mathrm{TeV}$ energies are sparse. The First G-APD Cherenkov Telescope (FACT) is a gamma-ray telescope dedicated to the long-term monitoring of bright $\mathrm{TeV}$ blazars. Operational since October 2011, it has collected more than three years of data from a dedicated sample of sources. The results of an automatic quick look analysis are publicly available on a website the same night. Based on this, other instruments are informed in case of a high flux state and target-of-opportunity observations are carried out. In the previous year, seven flare alerts have been sent to the community and several periods of strong variability have been observed for the blazars Mrk 421 and Mrk 501.

The 34th International Cosmic Ray Conference,

30 July- 6 August, 2015

The Hague, The Netherlands

*Speaker. 


\section{FACT - Ideal Monitoring Telescope}

The First G-APD Cherenkov Telescope (FACT) has been operational on the Canary Island of La Palma since October 2011. Details on the telescope and the status of the project are discussed in [1]. One of the major goals of the project is the long-term monitoring of bright $\mathrm{TeV}$ blazars. Thanks to the excellent performance [2] of the photosensors, FACT is an ideal instrument for monitoring observations.

\subsection{Enhanced Duty Cycle}

FACT is the first Cherenkov telescope to use silicon photosensors (SiPMs, a.k.a. Geigermode Avalanche Photodiodes (G-APDs)) in regular operation and has shown that they are a promising alternative to the commonly used photomultiplier tubes (PMTs) [2]. They have advantages that help enlarging the duty cycle of the instrument.

\subsubsection{Observations during Bright Moon}

The most obvious advantage is that SiPMs do not show any aging when exposed to bright light. While instruments using PMTs cannot operate during full moon or operate with reduced performance, FACT can operate under very bright light conditions as shown in [3]. As the telescope is operated remotely, it is for safety reasons allowed to operate only when somebody of the neigbouring telescopes is on the observatory site. Although this introduces few days without observations around full moon, the total observation time is enlarged by about one third and the size of the gaps around full moon is decreased. In the last twelve months, the telescope was operated for about 1960 hours. With a maximum of $65 \%$ of moon light, this would have been only 1380 hours, and observing only during dark time, i.e. no moon light and no twilight, the operation time would have been reduced to 1020 hours.

\subsubsection{Stable Detector Performance}

As the gain of the SiPMs depends on the applied voltage and the temperature, this has to be accounted for. Operating the camera in an environment with changing temperature and variable ambient light, the approach of keeping the gain stable by adapting the applied voltage has been chosen. Measuring the temperature and the currents in the sensors, the applied voltage is adapted such that the gain remains constant. Details on this feedback system can be found in [4] and [5], and the stable performance is also discussed in [6]. Keeping the gain stable, ensures a detector performance homogeneous in the camera and constant over time without the need of an external calibration device. For monitoring observations of variable sources over several years, a stable detector performance facilitates the analysis and helps obtaining consistent results.

\subsubsection{Remote and Automatic Operation}

In addition, a stable detector performance fosters remote and automatic operation. From June 2012, the telescope has been operated remotely. Thanks to the stability of the photosensors and the modular and reliable slow control software framework, the operation of the telescope could be automized [4]. As the data taking is performed automatically with a script that is running the complete night, manual interaction is only needed in case of unforseen events or bad weather. By 

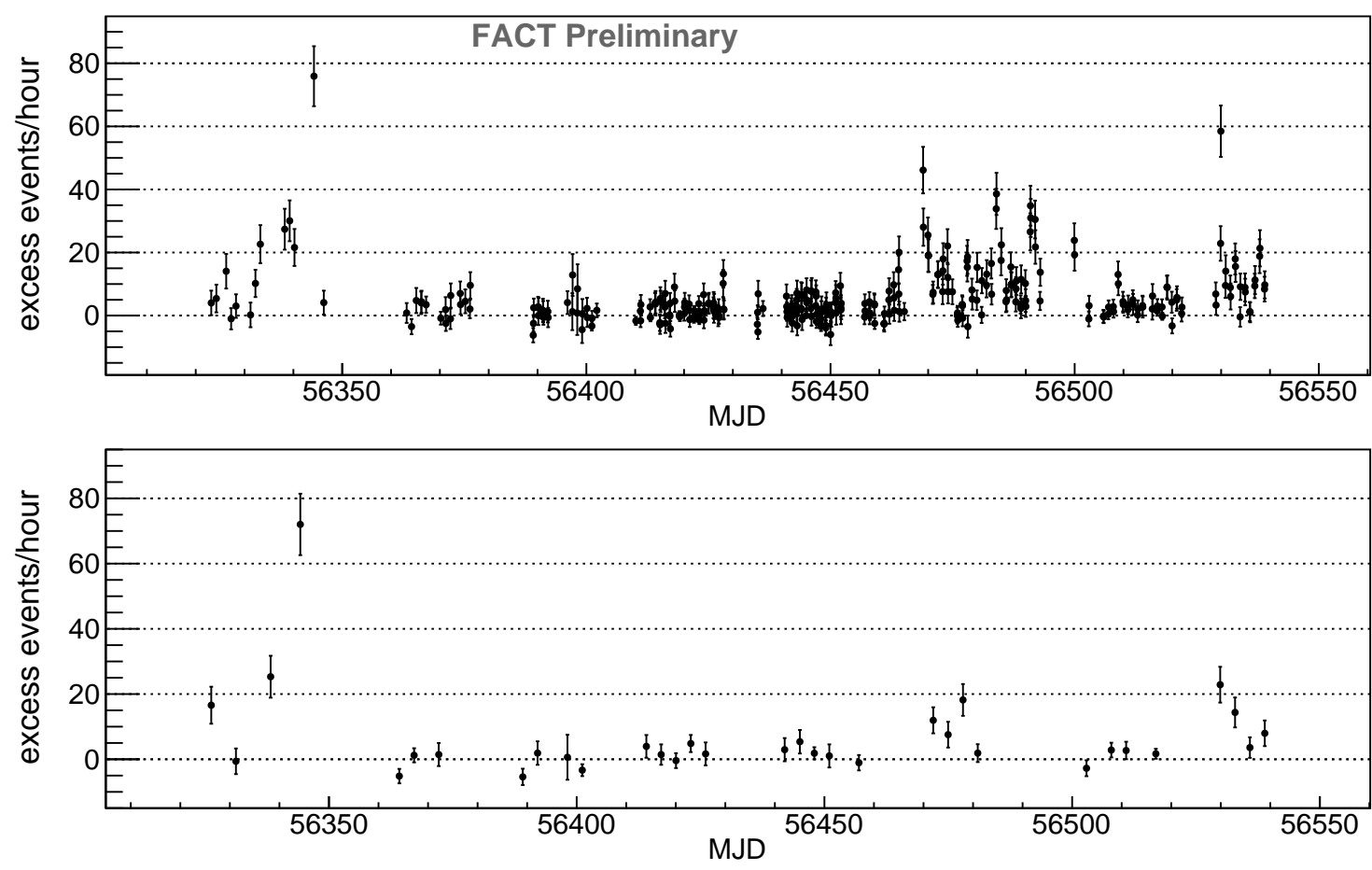

Figure 1: Comparison of excess rate curves from the long-term monitoring of FACT (upper plot) and the observation strategy with larger IACTs (lower plot). The plot has been done using the FACT data of Mrk 501 from the year 2013. The lower plot is also done with data from FACT but assuming only observations along the monitoring strategy of larger IACTs as described in the text. Each data point contains one hour of data.

increasing the data taking efficiency as shown in [1] and [5], the automation helps increasing the duty cycle of the instrument.

\subsection{Status of TeV Monitoring}

To study active galactic nuclei (AGN) at all time scales from minutes to years, a consistent and continous monitoring is mandatory. While for example in the optical regime a wide range of monitoring data are available thanks to the large number of instruments, the data samples at other wavebands are rather sparse. At TeV energies, for a long time only few sources were known and therefore the major goal of the large instruments is the detection of new sources and new source classes. As the importance of monitoring and multi-wavelength (MWL) observations is known, MWL campaigns are organized each year for some selected sources such as the blazars Mrk 421 and Mrk 501, e.g. [7] or [8]. However even within these campaigns, the data sample is rather sparse. Over a few months about every few nights about one hour of data are taken by one of the large imaging air Cherenkov telescopes (IACTs). With this sampling, not all time scales can be probed and information about the duration of flares is lost. Fig. 1 shows a comparison between the monitoring done by the large IACTs and the long-term monitoring done by FACT which is more continous and more dense sampled. The upper plot show the excess rate curve for Mrk 501 from FACT of the year 2013 in nightly bins. For comparison, the curve is reproduced 
with selection criteria mimicking the observation strategy of the large IACTs. This is shown in the lower plot, where the selection criteria were that there was less than 65 of moon light, only one hour of observation per night and one observation every third night. FACT on the other hand takes data whenever possible. To have best observations conditions, usually the sources are observed with a zenith distance smaller than $45^{\circ}$ unless in combination with a MWL campaign where the observation window might be extended to have overlapt with other instruments. Like this, a source is usually observed between one and six hours per night. This gives not only a denser sampling but also longer observation times per night providing better chances to catch the rise or decay of a flare and enlarging the window of accessible variability timescales. Naturally, the energy threshold of an observation increases with increasing zenith distance and increasing amount of ambient light.

As one can see in Fig. 1, with the observation strategy of the large IACTs not only some flaring activity remains undiscovered (between MJD 56465 and 56495 and around MJD 56530), but also information on the duration of a flare. For example if the flux is high in one night and low in the next, as around MJD 56345, this remains unnoticed in the less dense sampling. Like this, the FACT monitoring can provide useful additional information about the flares and probe additional time scales. Also the total amount of data of 411 hours from FACT observations is much larger than roughly 32 hours for a large IACT.

Another aspect is the unbiased sampling. As the large IACTs often react to flare alerts from other instruments, their data sample is biased towards higher fluxes and more variability. With FACT on the other hand, the sample is unbiased, as the telescope does continous and regular monitoring. Like this, all flux states are accessible, and the data can be used to study flare probability and the duty cycle of the sources.

\section{Quick Look Analysis}

To study short term variability and MWL behaviour during flares in more detail, it is important to trigger observations of larger IACTs and instruments at other wavelengths as quickly as possible. For this, a quick look analysis has been set up on site.

\subsection{Flare Alerts}

Since December 2012, an analysis is running on site which processes the data immediately once they are written on disk. The analysis is based on the software framework MARS [9], and the analysis steps are performed as described in [10]. As a result, excess rate curves are provided on a website [11]. This website has been publicly available since September 2013. During the night, the results go online immediately after processing. As of March 2014, alerts are sent, if the excess rate increases above a predefined value. In the last year, seven flare alerts have been sent. As follow-up of the first flare alerts for Mrk 501 in June 2014, a large outburst has been observed by several instruments in the night of 23rd to 24th of June [12].

\subsection{Results}

In Fig. 2, the excess rates from 3.5 years of monitoring for Mrk 421 and Mrk 501 are shown. The horizontal, dashed lines correspond to the start of a new year. For the presented result, no data check has been applied. Also, the excess rate does not include any correction for the effect of the 

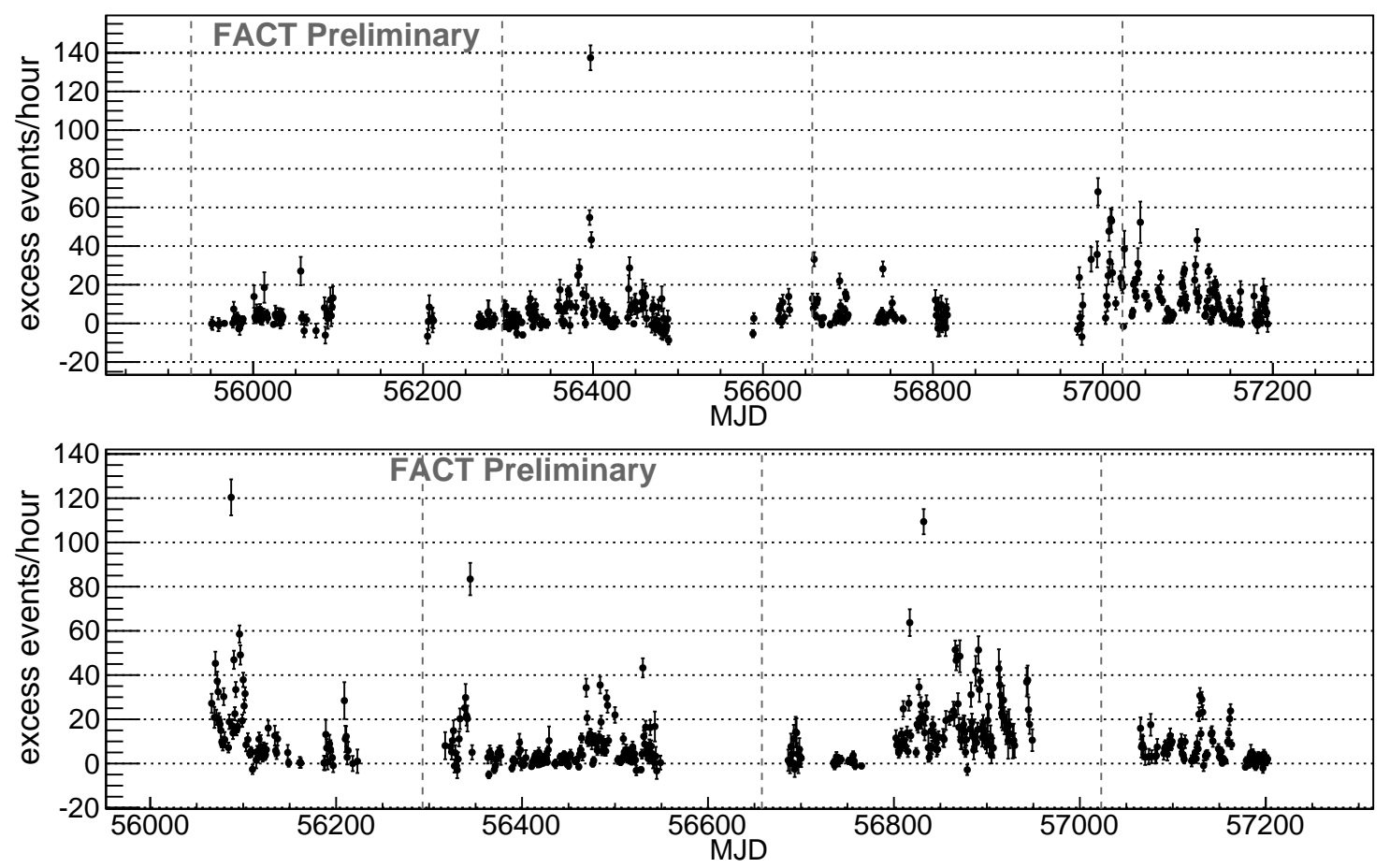

Figure 2: Excess rate curves from the long-term monitoring of FACT for the two sources Mrk 421 (upper plot) and Mrk 501 (lower plot). The horizontal, dashed lines correspond to the beginning of a new year.

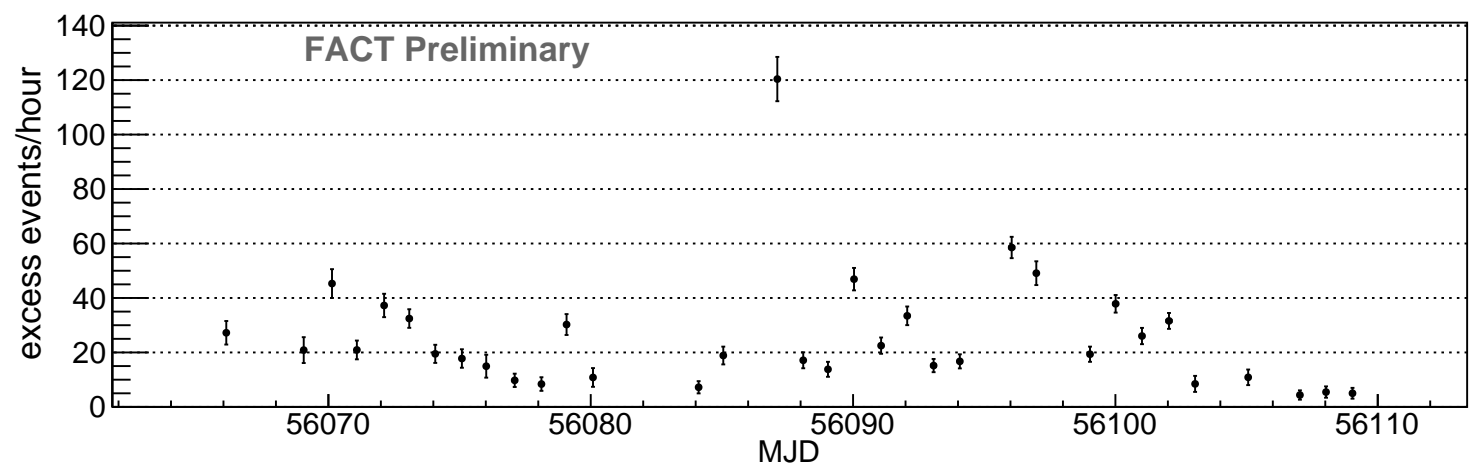

Figure 3: Excess rate curve from the flaring activity of Mrk 501 in June 2012. The plot covers the time range from mid May until end of June 2012.

zenith distance or trigger threshold, so that the displayed values correspond to a lower limit on the flux. Both sources show enhanced activity for few months during the total observation time.

For Mrk 421, the observations started in January 2012 and in April 2014, a bright flare was observed. In the flare night, the source was detected with a significance of about 14 sigma in 3.8 hours of observation.

The observations of Mrk 501 started in May 2012 during a MWL campaign when also some flaring activity took place. The source remained active in 2013 and 2014. 

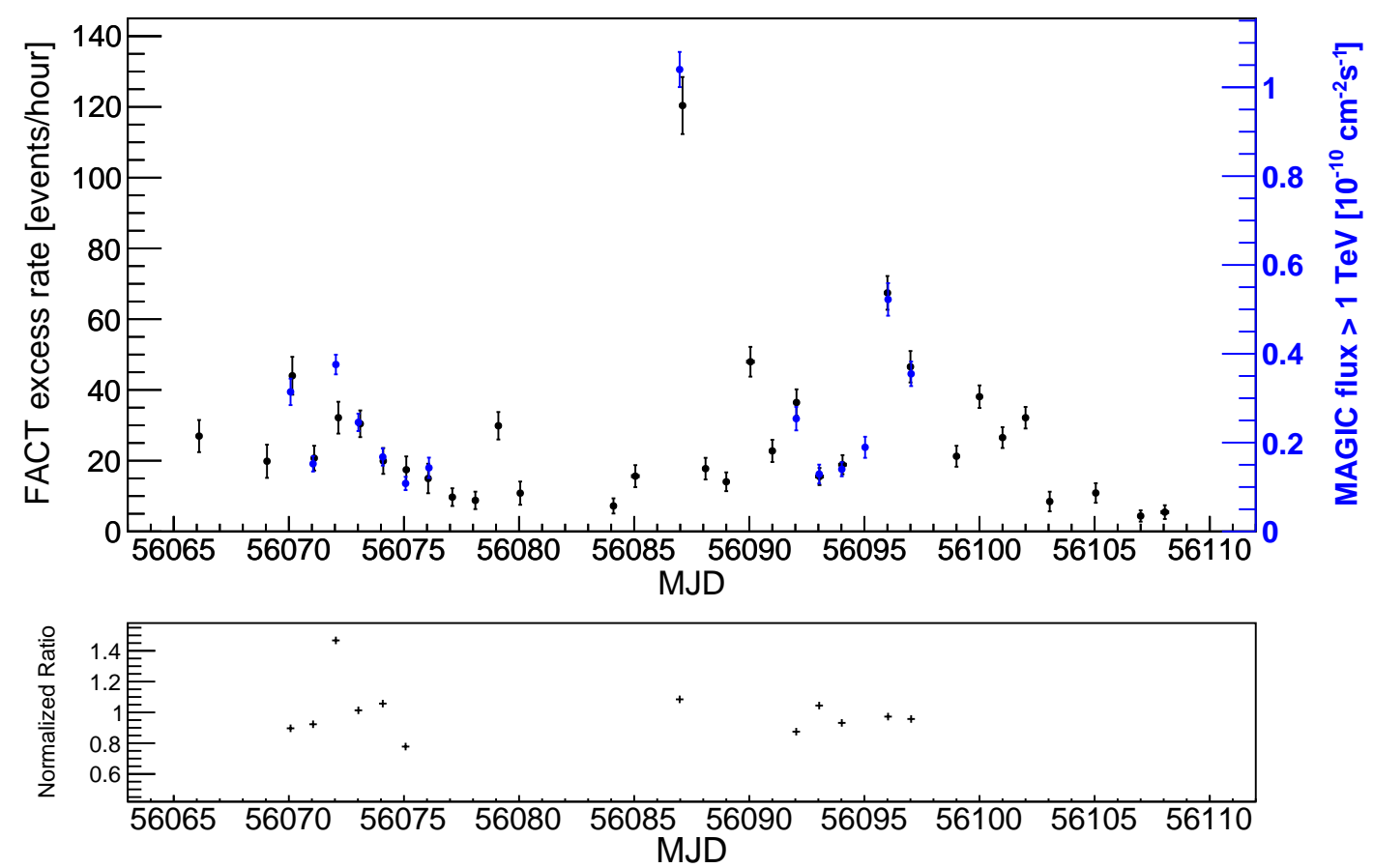

Figure 4: Comparison of the excess rate from FACT and the flux above $1 \mathrm{TeV}$ measured by MAGIC [14]. The upper panel shows the MAGIC flux in blue and the FACT excess rate in black, where the horizontal errorbars indicate the observation time. In the lower panel, the normalized ratio of the two measurements is shown for nights in which both instruments have overlapping observation time. But the data points do not only include strictly simultaneous data. Deviations may therefore come from intra-night variability.

\subsection{Correlation with MAGIC data}

A zoom to the flare in Jun 2012 is shown in Fig. 3. Between 18th of May and 30th of June, FACT observed Mrk 501 a data sample of 96 hours in 34 nights are taken into account, of which in 12 nights also MAGIC observed. The zenith distance in the FACT data sample was between $10^{\circ}$ and $60^{\circ}$ and covering all light conditions. In Fig. 4, a comparison with the MAGIC measurement is shown. In the upper plot, both measurements are shown: the FACT excess rate in black and the MAGIC flux above $1 \mathrm{TeV}$ in blue. While FACT observed in almost all nights, MAGIC had less and shorter measurements as the instrument has a very crowded schedule. The gap in the MAGIC data before the bright flare is due to the full moon where the MAGIC observation time is limited by the photosensors which suffer from aging when exposed to too much light. FACT, on the other hand, can still observe thanks to the SiPMs and therefore close the gap. Already end of May, the enhanced flux of the source was communicated to MAGIC. The big flare in the night from 8th to 9 th of June was observed by both instruments. In the lower panel, the normalized ratio of the two measurements is shown. For this, all nights were used in which both instruments have overlapping observation time. However, not only strictly simultaneous data were included. For the FACT data, the data check described in [13] has been applied. No selection on zenith distance and ambient light or correction for their effect on the energy threshold and therefore on the excessrate was performed. 
To get the normalization factor, the ratio has been plotted and fitted with a constant. Applying this normalization factor, the ratio nicely agrees with one showing that the two measurements agree. Slight deviation from one can come from intra-night variability or the above mentioned effect of the zenith distance and ambient light on the excess rate. Some of the nights show intra-night variablity can be observed. In the night with the largest deviation (MJD 56071), there is only 35 minutes of overlap in the observation where FACT covered a time of 3.6 hours and MAGIC two hours and the FACT excess rate shows intra-night variations by a factor of two. This can easily explain a factor of 1.5 between the measurements. Consequently, the FACT excess rates can nicely be used to further constrain the flare behaviour.

\section{Conclusion and Outlook}

As MWL and ToO observations are important, high priority has been put to the quick look analysis and to flare alerts. Since 2.5 years, the quick look analysis is running successfully in La Palma and since more than one year alerts are sent to the community.

The comparison of the FACT excess rate with the MAGIC light curve nicely shows that the results are consistent and can therefore be used. The work on fluxes from FACT are ongoing. As shown in [15], the spectrum of the Crab Nebula measured with FACT is consistent with the measurements of other instruments.

As the excess rate curves in Fig. 2 and Fig. 3 nicely show, flares can have a duration shorter than a few nights. There are several examples where the flux is low in the first night, high in the second night and low again in the third night. Still, there are cases where the rising and falling of the flux is not visible within the night. These parts of the flares are often not visible because of the gaps in the light curves due to daytime. Even with observations of six hours per night, 18 hours remain in which the flux is not know. To better catch also the rising and falling edge of a flare, more continuous monitoring is necessary, i.e. the gaps due to daytime have to be closed. To do so, the natural conclusion is to build more small monitoring telescopes around the globe [16].

In Mexico, there are two telescope mounts available. The goal of the project M@TE (Monitoring at $\mathrm{TeV}$ Energies) is to build a SiPM camera for one of the telescope and join the blazar monitoring [17]. Other possible sites, to close the gaps even more, are India, Japan and Hawaii. Given the dropping prices of the photosensors, these extensions for the long-term monitoring have become affordable small-scale instruments.

Acknowledgments The important contributions from ETH Zurich grants ETH-10.08-2 and ETH-27.121 as well as the funding by the German BMBF (Verbundforschung Astro- und Astroteilchenphysik) and HAP (Helmoltz Alliance for Astroparticle Physics) are gratefully acknowledged. We are thankful for the very valuable contributions from E. Lorenz, D. Renker and G. Viertel during the early phase of the project. We thank the Instituto de Astrofisica de Canarias allowing us to operate the telescope at the Observatorio del Roque de los Muchachos in La Palma, the Max-Planck-Institut für Physik for providing us with the mount of the former HEGRA CT 3 telescope, and the MAGIC collaboration for their support. Part of this work is supported by Deutsche Forschungsgemeinschaft (DFG) within the Collaborative Research Center SFB 876 "Providing Information by Research-Constrained Analysis", project C3. 


\section{References}

[1] A. Biland et al. (FACT Collaboration), FACT - Status and Experience from Three Years Operation of the First SiPM Camera, These proceedings ID 1032

[2] A. Biland et al. (FACT Collaboration), Calibration and performance of the photon sensor response of FACT - the first G-APD Cherenkov telescope JINST 9 (2014) P10012 [arXiv: 1403.5747 ]

[3] M.L. Knoetig et al. (FACT Collaboration), FACT - Long-term stability and observations during strong Moon light, ICRC 2013 [arXiv1307.6116]

[4] T. Bretz et al. (FACT Collaboration), FACT - Operation of the First G-APD Cherenkov Telescope, IEEE-RT 2014 [arXiv1407.1988]

[5] T. Bretz et al. (FACT Collaboration), FACT - The G-APD revolution in Cherenkov astronomy, IEEE-NSS/MIC 2013 [arXiv1403.3573]

[6] D. Neise et al. (FACT Collaboration), FACT - Performance of the First SiPM Camera, These proceedings ID 706

[7] A.A. Abdo et al., Fermi Large Area Telescope Observations of Markarian 421: The Missing Piece of its Spectral Energy Distribution, ApJ 736, 131 [arXiv:1106.1348]

[8] A.A. Abdo et al., Insights into the High-energy gamma-ray Emission of Markarian 501 from Extensive Multifrequency Observations in the Fermi Era, ApJ 727, 129 [arXiv: 1011.5260 ]

[9] T. Bretz and D. Dorner MARS - The Cherenkov Observatory Edition, AIPC, 1085

[10] D. Dorner et al. (FACT Collaboration), FACT - Monitoring Blazars at Very High Energies, Fermi Symposium 2014 [arXiv1502.02582]

[11] FACT Quick Look Analysis, http://www.fact-project.org/monitoring

[12] C. Stegmann et al., Increased VHE activity from Mrk 501 detected with H.E.S.S., Atel \#6268

[13] D. Dorner et al. (FACT Collaboration), FACT - Long-term Monitoring of Bright TeV-Blazars, ICRC 2013 [arXiv1311.0478]

[14] F. Borracci, U. Barres de Almeide, D. Paneque, J. Sitarek for the MAGIC Collaboration, MAGIC results and multi-wavelength observations of a Mrk 501 flare in June 2012 ICRC 2013 [arXiv1307.8344]

[15] F. Temme et al. (FACT Collaboration), FACT - First Spectrum from a SiPM Cherenkov Telescope, These proceedings ID 707

[16] M. Backes et al. (FACT Collaboration), Long-term monitoring of blazars - the DWARF network, ICRC 2009

[17] M. Gonzalez, R. Alfaro, G. Tovmasian, T. Bretz, D. Dorner, Private communication 